\title{
Effects of Trimebutine Maleate (TM-906) on the Spontaneous Contraction of Isolated Duodenum and Ileum in Both Guinea Pigs and Rabbits
}

\author{
Hideyuki TAKENAGA, Tetsuo MAGARIBUCHI and Hajime TAMAKI \\ Biological Research Laboratory. Tanabe Seiyaku Co., Ltd., \\ Toda, Saitama 335, Japan \\ Accepted September 13, 1985
}

\begin{abstract}
Effects of trimebutine maleate (TM-906) on the spontaneous contraction of isolated duodenum and ileum were studied in both guinea pigs and rabbits. In the duodenum and ileum of both guinea pigs and rabbits, TM-906 $\left(10^{-6} \mathrm{~g} / \mathrm{ml}\right.$, $10^{-5} \mathrm{~g} / \mathrm{ml}$ ) produced a potentiation of the spontaneous contraction in preparations with low contractile activity (low tone or small contraction), while it caused an inhibition of the spontaneous contraction in preparations with high contractile activity (high tone or large contraction). The potentiation of spontaneous contraction by TM-906 was more pronounced in the ileum than in the duodenum of both guinea pigs and rabbits. When the spontaneous contraction of duodenum and ileum was decreased by atropine, the potentiation of spontaneous contraction by TM-906 was further augmented and was more pronounced in the ileum than in the duodenum. When the spontaneous contraction was remarkably potentiated by physostigmine or acetylcholine. TM-906 markedly inhibited the potentiated spontaneous contraction, and the potentiation by TM-906 seen in preparations with low contractile activity disappeared. From these results, it is concluded that TM-906 produces, depending on the contractile activity of the preparations, a potentiation or an inhibition of the spontaneous contraction of duodenum and ileum in both guinea pigs and rabbits and that the potentiation by TM-906 is more pronounced in the ileum than in the duodenum. It is suggested that the endogeneous acetylcholine partly modifies the effects of TM-906, but that it does not relate to the more pronounced potentiation by TM-906 in the ileum than in the duodenum.
\end{abstract}

Trimebutine maleate (TM-906) is used in the treatment of gastrointestinal disorders including abdominal pain, dyspepsia, irritable bowel syndrome and post-operative ileus. The efficacies of TM-906 in spastic colon $(1,2)$ and in recovery of the digestive transit time after abdominal surgery (3) were supposed to due to the inhibitory and stimulatory effects of TM-906 on intestinal motility. It has been reported that TM-906 produces an excitation of the spontaneous contraction of the isolated guinea pig colon in preparations with low tone, while it causes an inhibition of the spontaneous contraction in preparations with high tone, and it was suggested that these effects of TM-906 are due to its direct action on smooth muscles (4). The present experiments were performed to study whether similar phenomena were observed in the duodenum and ileum of both guinea pigs and rabbits.

\section{Materials and Methods}

Male guinea pigs (300-400 g) and male albino rabbits $(2.0-3.0 \mathrm{~kg})$ were stunned and bled. The duodenum $(2 \mathrm{~cm}$ caudad to the pyrolus) and the ileum (10 $\mathrm{cm}$ orad to the ileocaecal junction) of both guinea pigs and rabbits were isolated and emptied of their contents, and longitudinal segments of 
$1 \mathrm{~cm}$ length were prepared. The preparations were suspended in an organ bath containing $15 \mathrm{ml}$ of modified Krebs solution, and isometric contraction was recorded on an inkwriting oscillograph through a strain gauge transducer. The initial tension applied to the muscle was adjusted to $0.2-1.0 \mathrm{~g}$ in guinea pigs and $0.5-5.0 \mathrm{~g}$ in rabbits, and the tone and amplitude of spontaneous contraction of the preparations mostly corresponded with the initial tension. As the effect of TM-906 at the second application became weaker than that at the first application, a fresh preparation was used each time the effect of TM-906 was studied. The composition of modified Krebs solution was as follows $(\mathrm{mM})$ : $\mathrm{NaCl}, 141.9 ; \mathrm{KCl}, 5.6$; $\mathrm{CaCl}_{2}$, 2.2; $\mathrm{MgCl}_{2}, 0.4 ; \mathrm{NaH}_{2} \mathrm{PO}_{4}, 0.4$; $\mathrm{NaHCO}_{3}, 11.9$ and glucose, 5.5. The solution was aerated with $95 \% \quad \mathrm{O}_{2}$ and $5 \% \quad \mathrm{CO}_{2}$.
Experiments were carried out at $37 \pm 1^{\circ} \mathrm{C}$. The following compounds were used: TM-906 (Tanabe Seiyaku Co., Ltd.), atropine sulfate (Wako Pure Chem. Ind., Ltd.), acetylcholine chloride and physostigmine salicylate (Nakarai Chem., Ltd.).

\section{Results}

\section{Guinea pig}

1-1. Effects of TM-906 on the spontaneous contraction of duodenum and ileum: Figures 1 and 2 show the effects of TM-906 on the spontaneous contraction of duodenum and ileum. In preparations of duodenum with low tone. TM-906 $\left(10^{-6} \mathrm{~g} / \mathrm{ml}, 10^{-5} \mathrm{~g} / \mathrm{ml}\right)$ caused a transient decrease of the amplitude of spontaneous contraction and then potentiated the amplitude of spontaneous contraction. TM-906 at the concentration of $10^{-5} \mathrm{~g} / \mathrm{ml}$ augmented the amplitude of
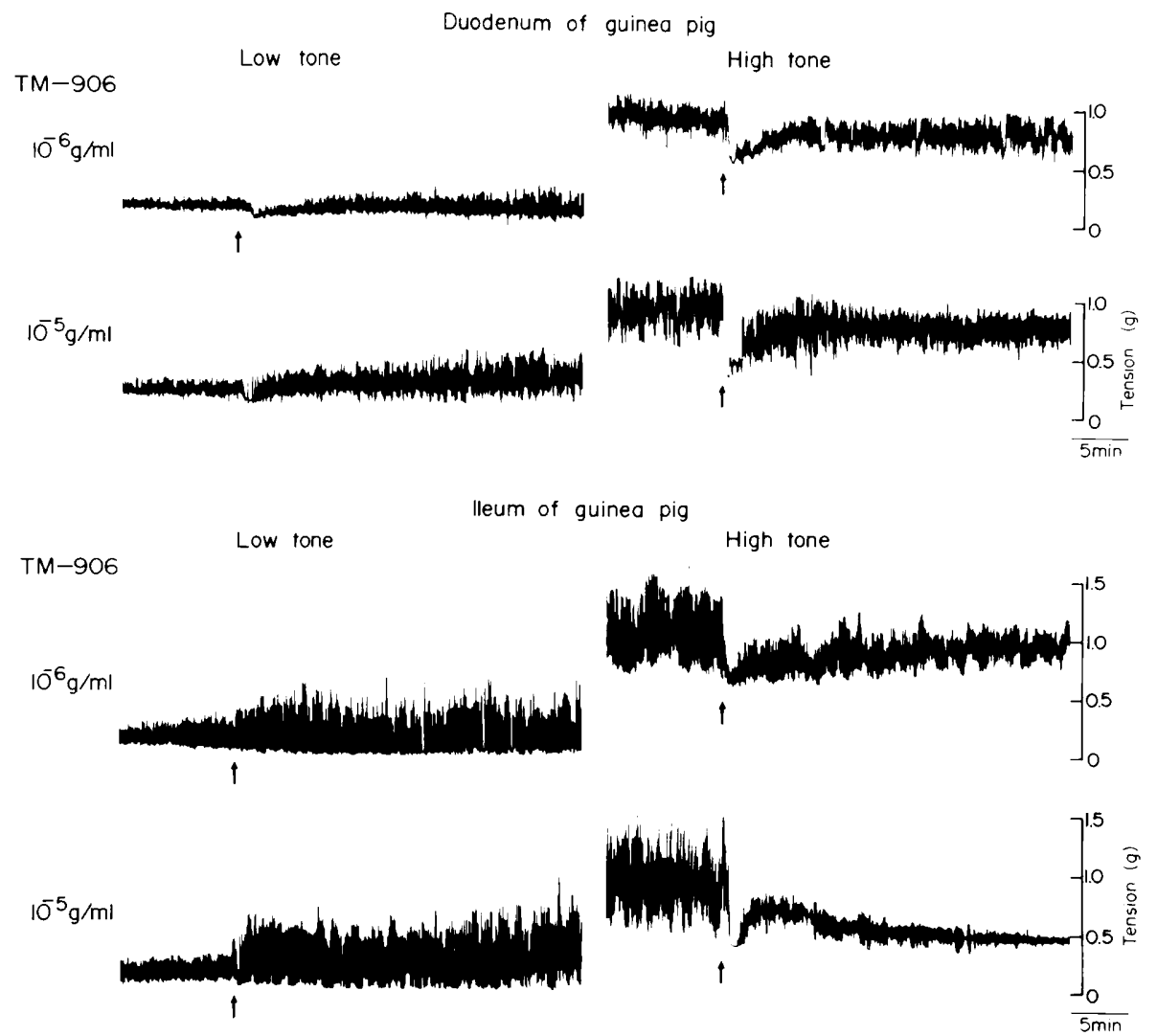

Fig. 1. Effects of TM-906 $\left(10^{-6} \mathrm{~g} / \mathrm{ml}, 10^{-5} \mathrm{~g} / \mathrm{ml}\right)$ on the spontaneous contraction of guinea pig duodenum and ileum in the preparation with low tone and that with high tone. 


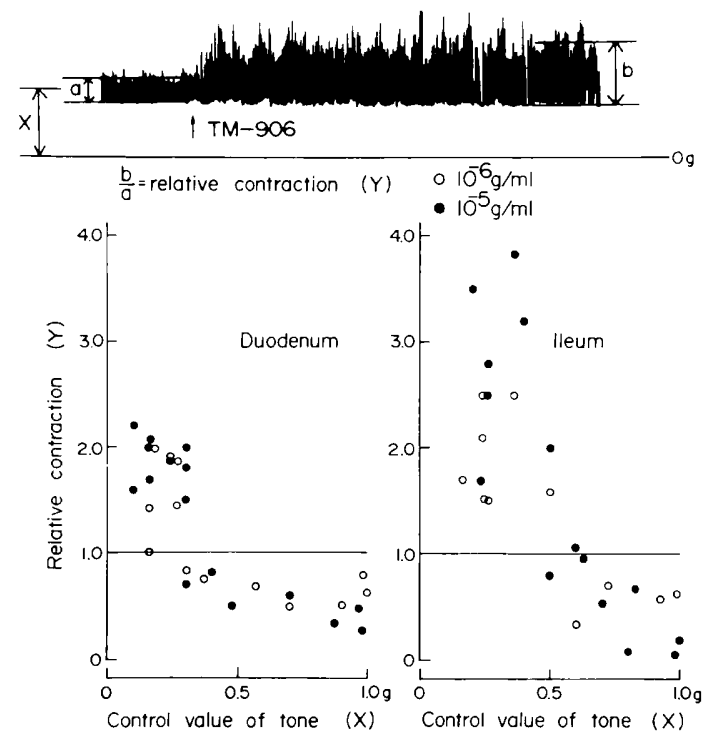

Fig. 2. Relationship between the effects of TM-906 $\left(10^{-6} \mathrm{~g} / \mathrm{ml}\right)$ on the amplitude of spontaneous contraction of guinea pig duodenum and ileum and control values of the tone. The values were obtained at 30 min after application of TM-906. Ordinate: relative contraction. Abscissa: control value of tone. The tone was estimated by measuring from $0 \mathrm{~g}$ to the value of the half amplitude of the spontaneous contraction as shown in the upper trace. The amplitude of spontaneous contraction was determined by visually measuring the mean amplitude.

spontaneous contraction approx. 1.9-fold (Fig. 7). In preparations of duodenum with high tone. TM-906 $\left(10^{-6} \mathrm{~g} / \mathrm{ml}, 10^{-5} \mathrm{~g} / \mathrm{ml}\right)$ decreased the tone and amplitude of spontaneous contraction. In the ileum, TM-906 $\left(10^{-6} \mathrm{~g} / \mathrm{ml}, 10^{-5} \mathrm{~g} / \mathrm{ml}\right)$ enchanced the amplitude of spontaneous contraction in preparations with low tone, while it decreased the tone and amplitude of spontaneous contraction in preparations with high tone (Figs. 1 and 2). In preparations with low tone, $10^{-5} \mathrm{~g} / \mathrm{ml}$ TM -906 augmented the amplitude of spontaneous contraction of ileum approx. 2.6-fold, and the potentiation by TM-906 was more pronounced in the ileum than in the duodenum (Fig. 7A). Application of $10^{-5} \mathrm{~g} / \mathrm{ml}$ TM-906 immediately caused a transient decrease of the amplitude of spontaneous contraction in preparations of ileum with low tone before the potentiation by TM-906 appeared.

1-2. Effects of TM-906 on the spontaneous contraction of the duodenum and ileum under treatment with atropine, physostigmine and acetylcholine: When atropine $\left(10^{-6} \mathrm{~g} / \mathrm{ml}\right)$ was added to the bathing solution, the tone and amplitude of spontaneous contraction of the duodenum and ileum remarkably decreased, and the application of $10^{-5} \mathrm{~g} / \mathrm{ml}$ TM-906 augmented the amplitude of spontaneous contraction approx. 3.0-fold in the duodenum and approx. 7.6-fold in the ileum (Figs. 3 and 7A). The potentiation by TM-906 was more significant in comparison with the control without atropine and more pronounced in the ileum than in the duodenum. When physostigmine $\left(10^{-7} \mathrm{~g} / \mathrm{ml}\right)$ or acetylcholine $\left(3 \times 10^{-7} \mathrm{~g} / \mathrm{ml}\right)$ was added to preparations of ileum with low tone, the low tone markedly increased and TM-906 remarkably decreased the increased tone, and the potentiation of amplitude of spontaneous contraction by TM-906 seen in preparations with low tone was not observed.

\section{Rabbit}

2-1. Effects of TM-906 on the spontaneous contraction of duodenum and ileum: In preparations of duodenum with small amplitude of spontaneous contraction (small contraction), TM-906 at the concentration of $10^{-6} \mathrm{~g} / \mathrm{ml}$ produced a slight inhibition followed by a gradual augmentation of the amplitude of spontaneous contraction (Figs. 4 and 5). In preparations of duodenum with large amplitude of spontaneous contraction (large contraction), TM-906 (10-6 $\mathrm{g} / \mathrm{ml})$ inhibited the amplitude of spontaneous contraction. In the ileum, TM-906 $\left(10^{-6} \mathrm{~g} / \mathrm{ml}\right)$ potentiated the amplitude of spontaneous contraction in preparations with small contraction, while it decreased the amplitude of spontaneous contraction in preparations with large contraction (Figs. 4 and 5). In preparations with small contraction. TM-906 $\left(10^{-6} \mathrm{~g} / \mathrm{ml}\right)$ potentiated the amplitude of spontaneous contraction approx. 1.4-fold in the duodenum and approx. 1.9-fold in the ileum (Fig. 7A). The potentiation by TM-906 was more pronounced in the ileum than in the duodenum.

2-2. Effects of TM-906 on the spon- 


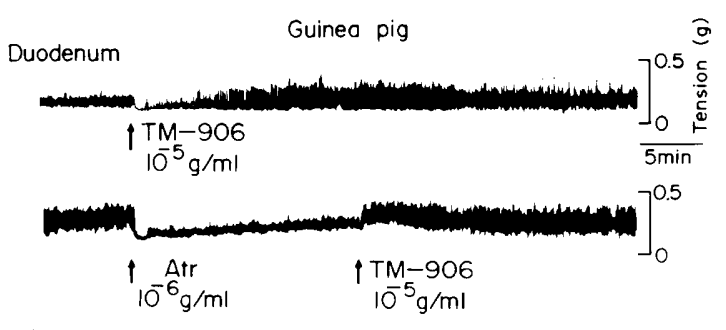

lleum
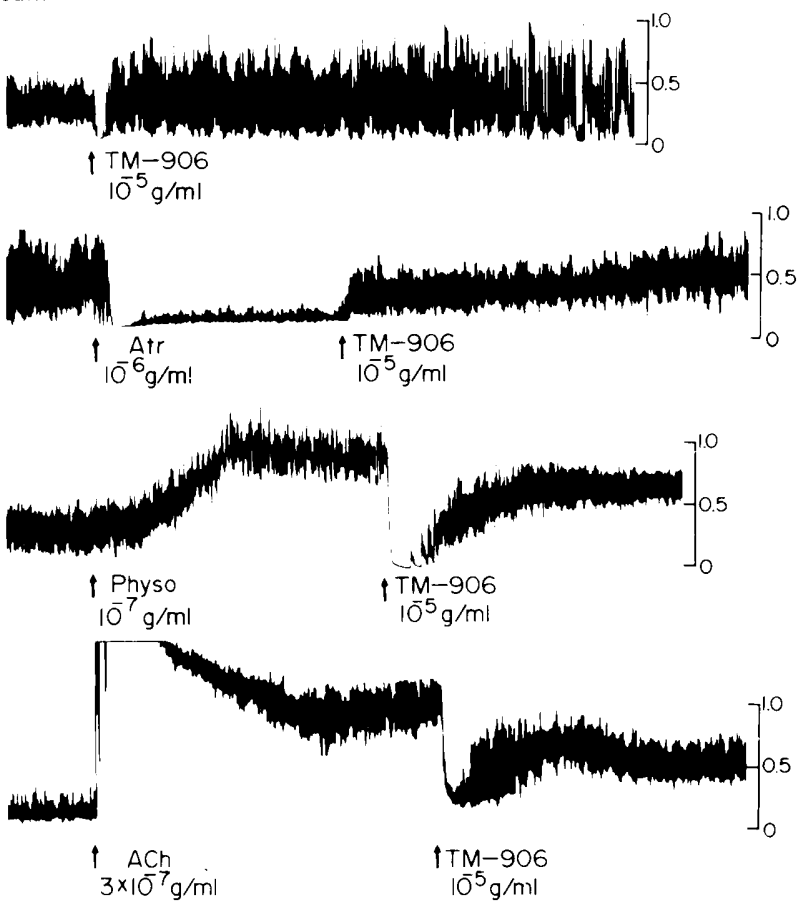

Fig. 3. Effects of TM-906 $\left(10^{-5} \mathrm{~g} / \mathrm{ml}\right)$ on the spontaneous contraction of guinea pig duodenum and ileum under treatment with atropine $\left(10^{-6} \mathrm{~g} / \mathrm{ml}\right.$. Art $)$, physostigmine $\left(10^{-7} \mathrm{~g} / \mathrm{ml}\right.$. Physo) or acetylcholine $\left(3 \times 10^{-7} \mathrm{~g} / \mathrm{ml}, \mathrm{ACh}\right)$.

taneous contraction of duodenum and ileum under treatment with atropine: When atropine $\left(10^{-6} \mathrm{~g} / \mathrm{ml}\right)$ was added to the bathing solution, the amplitude of spontaneous contraction of duodenum and ileum remarkably decreased, and application of $10^{-6} \mathrm{~g} / \mathrm{ml}$ TM-906 gradually potentiated the amplitude of spontaneous contraction approx. 1.8-fold in the duodenum and 2.4-fold in the ileum (Figs. 6 and 7B). The potentiation by TM906 was more significant in comparison with the control without atropine and was more pronounced in the ileum than in the duodenum.

\section{Discussion}

Regarding the spontaneous contraction of the duodenum and ileum of both guinea pig and rabbit, TM-906 $\left(10^{-6} \mathrm{~g} / \mathrm{ml}, 10^{-5} \mathrm{~g} / \mathrm{ml}\right)$ potentiated the amplitude in preparations with low contractile activity (low tone or small contraction). while it decreased the tone and amplitude in preparations with high contractile activity (high tone or large contraction). This difference of the responses to TM-906 depending on the contractile activity of the preparations were the same as those observed in the isolated guinea pig colon (4). The potentiation of spontaneous contraction 
Robbit

Duodenum

lleum

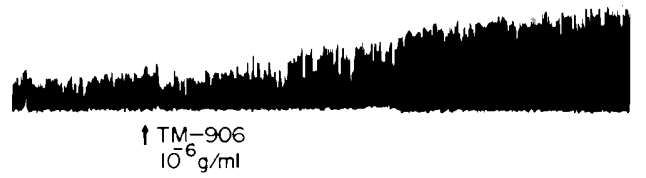

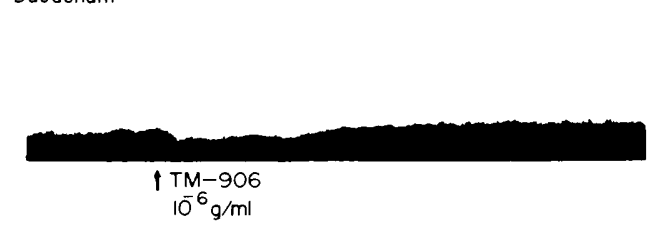

Small contraction

Fig. 4. Effects of TM-906 $\left(10^{-6} \mathrm{~g} / \mathrm{ml}\right)$ on the spontaneous contraction of rabbit duodenum and ileum in the preparation with small amplitude of spontaneous contraction (small contraction) and that with large amplitude of spontaneous contraction (large contraction).

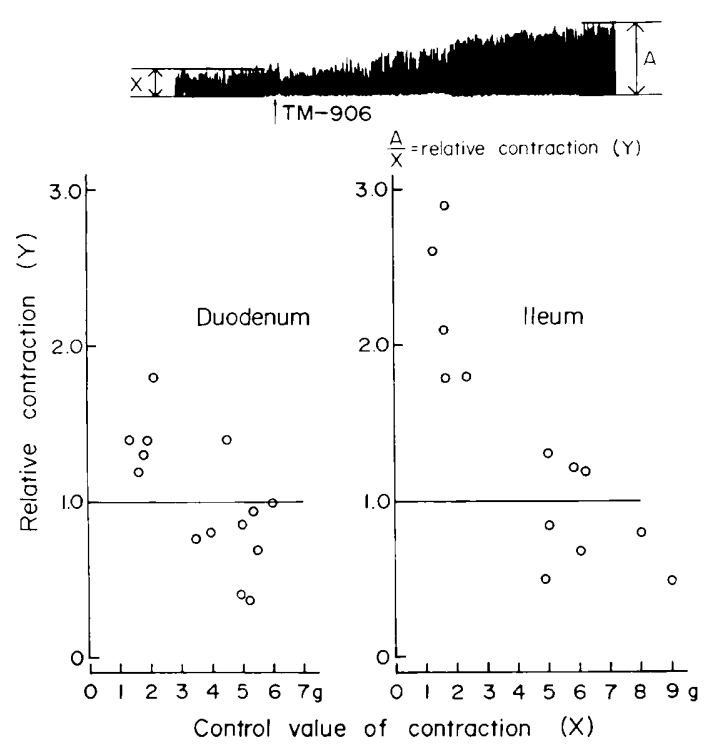

Fig. 5. Relationship between the effects of TM-906 on the amplitude of spontaneous contraction of rabbit duodenum and ileum and control values of the amplitude of spontaneous contraction. The values were obtained at $30 \mathrm{~min}$ after application of TM-906. For other details, see Fig. 2.

by TM-906 in both guinea pig and rabbit was more pronounced in the ileum than in the duodenum.

As shown in Figs. 3 and 6 , atropine markedly decreased the tone and amplitude of spontaneous contraction of the duodenum and ileum in both guinea pig and rabbit, and physostigmine, an agent known to increase endogeneous acetylcholine levels, and acetylcholine markedly increased the tone of the guinea pig ileum preparation. The potentiation of the amplitude of spontaneous contraction by TM-906 was further augmented under treatment with atropine, while the potentiation of spontaneous contraction by TM-906 disappeared when the tone was markedly increased by physostigmine or acetylcholine. These observations indicate that endogeneous acetylcholine contributes to the tone and amplitude of spontaneous contraction and modifies the effects of TM906. Therefore, the effects of TM-906 under treatment with atropine was measured by way of relative contraction. As shown in Fig. 7. the potentiation of spontaneous contraction by TM-906 was more pronounced in the ileum than in the duodenum in the absence or presence of atropine. It is suggested that the larger reaction to TM-906 in the ileum than in the duodenum is not related to the endogeneous acetylcholine.

TM-906 produced an inhibition of the spontaneous contraction of the duodenum and ileum with high contractile activity and it has been previously reported that under 


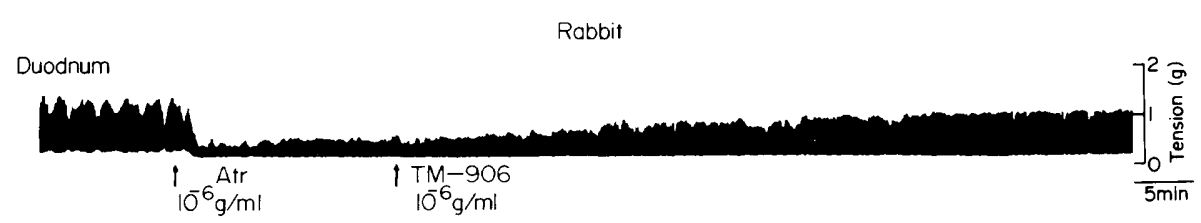

lleum

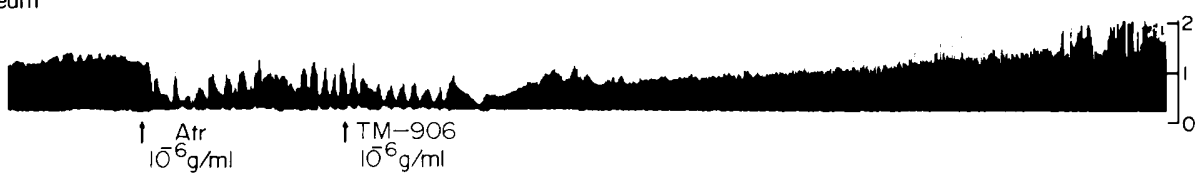

Fig. 6. Effects of $T M-906\left(10^{-6} \mathrm{~g} / \mathrm{ml}\right)$ on the spontaneous contraction of rabbit duodenum and ileum under treatment with atropine $\left(10^{-6} \mathrm{~g} / \mathrm{ml}\right.$, Art $)$.

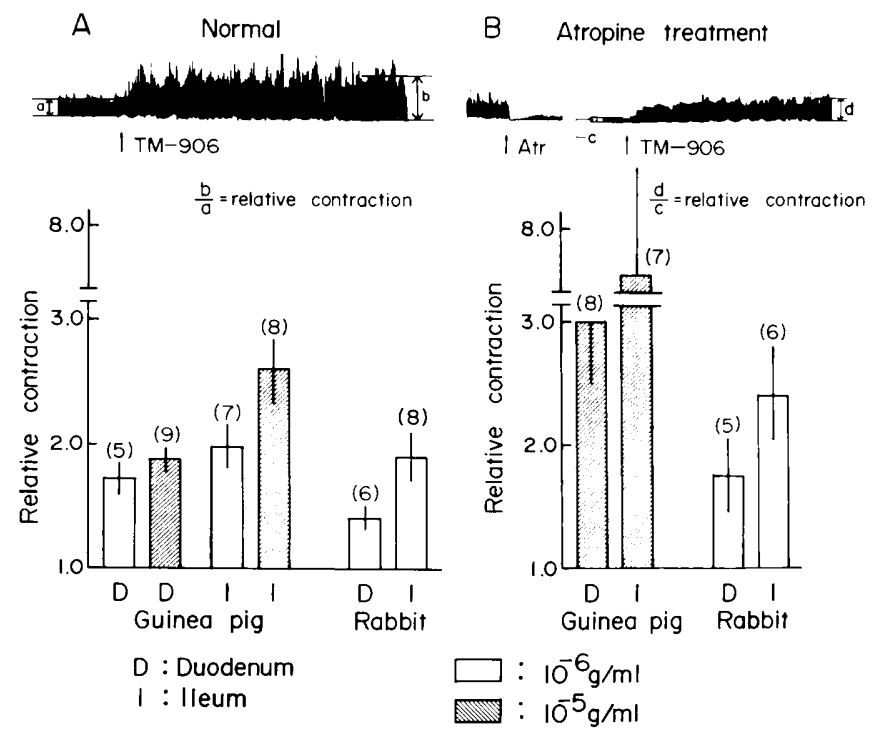

Fig. 7. The potentiation of the amplitude of spontaneous contraction of duodenum and ileum by TM906 in preparations of both guinea pigs and rabbits with low tone or small contraction in the absence $(A)$ or presence (B) of atropine $\left(10^{-6} \mathrm{~g} / \mathrm{ml}\right.$. Atr). The values were obtained from preparations in which TM-906 potentiated the spontaneous contraction. The figures in the parentheses indicate the number of animals. The vertical bars indicate the S.E. of the mean. For other details, see Fig. 2.

treatment with tetrodotoxin. TM-906 itself evokes the contractile response of the guinea pig gallbladder and colon, while it inhibits the contractile response to $\mathrm{KCl}$ (4, 5). Therefore, TM-906 has a potentiating effect in addition to an inhibitory effect in various organs of gastrointestinal tracts, and these effects are due to direct actions on the smooth muscles. In preparations of the duodenum and ileum with low contractile activity, TM-906 potentiated the spon- taneous contraction. Therefore, the inhibitory effect of TM-906 may be masked by the potentiating effect in these preparations.

Furukawa and Kimoto found that TM-906 depolarized the cell membrane in the guinea pig antrum (6). TM-906 would depolarize the cell membrane in the duodenum and ileum as well as antrum and would increase the transmembrane influx of $\mathrm{Ca}^{++}$. This assumption is supported by the result that TM-906 potentiates the spontaneous con- 
traction in prepartions with low contractile activity.

Furukawa and Kimoto also found that TM-906 did not further depolarize the cell membrane in the guinea pig antrum depolarized partially by $\mathrm{KCl}(11.8-47.2 \mathrm{mM})$ (6). It is well known that acetylcholine depolarizes the cell membrane in visceral smooth muscles (7). Endogeneous acetylcholine partly contributes to the spontaneous contraction of duodenum and ileum as described above, and the contribution is thought to be more considerable in preparations with high contractile activity than those with low contractile activity. Namely, it can be assumed that the cell membrane potential in preparations with high contractile activity is decreased by endogeneous acetylcholine in comparison with that in preparations with low contractile activity. In these preparations with decreased membrane potential, TM-906 may cause less or no depolarization of the cell membrane similarly to the results reported in the cell membrane in the guinea pig antrum depolarized partially by $\mathrm{KCl}(6)$, and hardly produces the potentiation of spontaneous contraction. It has been reported that TM-906 inhibits $\mathrm{CaCl}_{2}$-induced contraction of the guinea pig gallbladder having the cell membrane depolarized fully by $100 \mathrm{mM} \mathrm{KCl}$ due to an inhibition of transmembrane influx of $\mathrm{Ca}^{++}(5)$. It is possible that in preparations with high contractile activity. only the inhibition of transmembrane influx of $\mathrm{Ca}^{++}$by TM-906 appears and TM-906 inhibits the spontaneous contraction.

As described above, it is speculated that TM-906 has an inhibitory effect due to the blockade of the transmembrane influx of $\mathrm{Ca}^{++}$ and also a potentiating effect due to the increase in transmembrane influx of $\mathrm{Ca}^{++}$ resulting from the depolarization of the cell membrane, and that the blockade of transmembrane influx of $\mathrm{Ca}^{++}$appears independently of the contractile activity. while the increase in transmembrane influx of $\mathrm{Ca}^{++}$is limited by the rnembrane potential. Namely, TM-906 does not produce an increase in transmembrane influx of $\mathrm{Ca}^{++}$in preparations with low membrane potential because TM906 causes no further depolarization of the cell membrane. Therefore, the following explanation can be made that in preparations with low contractile activity which have a high membrane potential, the increase in the transmembrane influx of $\mathrm{Ca}^{++}$resulting from depolarization of the cell membrane by TM906 acts stronger than the blockade of transmembrane influx of $\mathrm{Ca}^{++}$, while in preparations with high contractile activity, the membrane potential is low and only the blockade of the transmembrane influx of $\mathrm{Ca}^{++}$ by TM-906 appears itself. The suggestion that TM-906 has an inhibitory effect due to the blockade of the transmembrane influx of $\mathrm{Ca}^{++}$and a potentiating effect due to the increase in the transmembrane influx of $\mathrm{Ca}^{++}$ is supported by the contradictory finding of Furukawa and Kimoto that the inhibition of spontaneous contraction of the guinea pig antrum by TM-906 was accompanied with a depolarization of the cell membrane (6).

In summary, it is concluded that TM-906 produces, depending on the contractile activity of the preparations, a potentiation or an inhibition of the spontaneous contraction of duodenum and ileum in both guinea pigs and rabbits and that the potentiation by TM906 is more pronounced in the ileum than in the duodenum. It is suggested that the endogeneous acetylcholine modifies the effects of TM-906, but that it does not relate to the more pronounced potentiation by TM-906 in the ileum than in the duodenum.

Acknowledgement: We express our gratitude to Dr. H. Nakajima, Director of the Biological Research Laboratory. Tanabe Seiyaku Co., Ltd. for constant encouragement, Dr. M. Takaiti for his helpful discussions and Mrs. K. Sato for typing this manuscript.

\section{References}

1 Lüttecke, K.: A trial of trimebutine in spastic colon. J. Int. Med. Res. 6, 86-88 (1978)

2 Moshal, M.G. and Herron, M.: A clinical trial of trimebutine (mebutin) in spastic colon. J. Int. Med. Res. 7, 231-234 (1979)

3 Malavaud, A.: Trial of trimebutine in the recovery of digestive transit in abdominal surgery (apropos of 100 cases). Anesth. Anal. 29, 65-69 (1972)

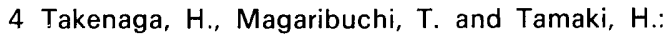
Effects of trimebutine maleate (TM-906) on the spontaneous contraction of isolated guinea pig colon. Japan. J. Pharmacol. 34, 177-181 (1984) 
5 Takenaga, H., Magaribuchi, T. and Tamaki, H.: Effects of trimebutine maleate (TM-906) on the smooth muscles of isolated guinea pig gallbladder. Japan. J. Pharmacol. 35, 439-443 (1984)

6 Furukawa, K. and Kimoto, Y.: Effects of trimebutine maleate (TM-906) on electrical and mechanical activities of smooth muscles of the guinea-pig stomach. Japan. J. Pharmacol. 35, 307-317 (1984)

7 Burnstock, G.: The effects of acetylcholine on membrane potential, spike frequency, conduction velocity and excitability in the taenia coli of the guinea pig. J. Physiol. (Lond.) 143, 165-182 (1958) 